\title{
Potential impact of combined influenza and pneumococcal vaccines on the severity of respiratory illness in COVID-19 infection among type 2 diabetic patients
}

\author{
Amr Shaaban Hanafy ${ }^{1}$ [ $\cdot$ Waseem M. Seleem ${ }^{1} \cdot$ Hany A. Elkattawy ${ }^{2,3}$
}

Received: 2 February 2021 / Accepted: 20 December 2021 / Published online: 22 January 2022

(c) The Author(s), under exclusive licence to Springer Nature Switzerland AG 2022

\begin{abstract}
To retrospectively assess the impact of regular yearly administration of recombinant influenza and single administration of pneumococcal conjugate vaccines on the occurrence of serious respiratory infection including COVID-19 in patients with type 2 diabetes mellitus. Hundred patients with type 2 diabetes mellitus were given Vaxigrip and Prevnar $13^{\circledR}$ vaccines and were evaluated by comprehensive clinical review, airflow screening questionnaire, and routine laboratory investigations with follow-up during the COVID-19 pandemic and compared to a control group of diabetic patients with the same inclusion criteria $(n=100)$. After Vaxigrip and Prevnar13, there is a significant improvement in respiratory symptoms and a decrease in the airflow screening questionnaire $(p=0.0001)$ with a significant improvement in inflammatory parameters as neutrophillymphocyte ratio, ESR, CRP, and platelet count. Four patients had mild COVID-19 (4\%), mainly gastrointestinal with no complications. Twenty-one out of 32 (65.6\%) patients in the control group had severe COVID-19. The hazard ratios of significant respiratory tract infection and death due to COVID-19 were 2.29 and 10.24 in the non-vaccinated control $(p=0.001)$. The severity of COVID-19 in diabetes correlated with HBA1C $(p=0.007)$, combined Vaxigrip and Prevnar13 vaccination $(p=0.0001)$, serum creatinine $(p=0.001)$, neutrophil-lymphocyte ratio $(p=0.001)$, and thrombocytopenia $(p=0.003)$. The present study suggested that the combination of Prevnar13 and Vaxigrip may be related to decreased occurrence of serious respiratory infections including COVID-19. Further randomized control trials may be needed to establish a direct causation between the two and clarify these associations.
\end{abstract}

Keywords Influenza $\cdot$ Pneumococcal vaccines $\cdot$ COVID-19 $\cdot$ Diabetes mellitus

\section{Introduction}

Newly developed viral epidemics put a significant and challenging economic burden on healthcare systems. The potency of the vaccine is determined by disease control, which relies on the age and immunocompetence of the patient [1].

Amr Shaaban Hanafy

dr_amr_hanafy@yahoo.com

1 Internal Medicine Department, College of Medicine, Zagazig University, Zagazig-40-Mostafa Fouad Street, Zagazig, Al-Sharkia 44519, Egypt

2 Department of Basic Medical Sciences, College of Medicine, Almaarefa University, Ad Diriyah, Saudi Arabia

3 Medical Physiology Department, Zagazig University, College of Medicine, Zagazig, Egypt
Previous experience in SARS-CoV-infected patients demonstrated a significant innate immune response as a cause of SARS-CoV-mediated disease. Neutralizing antibody response to SARS-CoV spike (S) glycoprotein is protective, including memory B cells, but it is short-lived in cured patients. Severe depletion of $\mathrm{T}$ cells had been demonstrated during the acute stage of this illness [2].

Lymphopenia is a laboratory feature of severity [3] with a substantial decline in CD4 + T, CD8 + T, natural killer cells (NC), and B cell lymphocytes as the most critical characteristics of immune dysfunction in COVID-19 [4]. It was explained by direct infection of $\mathrm{T}$ cells and macrophages through ACE2 receptor-mediated viral entry, the aggressive inflammatory release of cytokine-favored $\mathrm{T}$ cell exhaustion, spleen, and lymph node invasion by the virus, and lactic acidemia that might occur in sepsis and cause inhibition to lymphocyte proliferation $[5,6]$. 
$\mathrm{CD} 8+\mathrm{T}$ cell reaction can surpass $\mathrm{CD} 4+\mathrm{T}$ cell reaction with high expression of CD69, CD38, and CD44 and enhanced expression of OX40 (CD134) and OX40L (CD252); when compared to healthy subjects, the latter is one of TNFR/TNF family members which can stimulate T cells promoting sustained $\mathrm{T}$ cell clonal expansion and survival making it a promising target for enhancing protection against viral infection [7].

In $O X 40-/-$ mice, $\mathrm{CD} 4+\mathrm{T}$ cell proliferation and the number of IFN- $\gamma$-producing $\mathrm{CD} 4+$ and number of $\mathrm{CD} 4+\mathrm{T}$ cells infiltrating the lungs of influenza virusinfected $O X 40-/-$ mice was reduced [8].

With the progression of COVID-19, lymphocyte dysfunction could be represented with the exhaustion of T lymphocyte and NK cells with upregulation of programmed cell death protein-1 (PD1), T cell immunoglobulin domain, and killer cell lectin-like receptor subfamily $\mathrm{C}$ member 1 (NKG2A) [9].

Neutrophils and neutrophil/lymphocyte ratio are considerably higher as lymphocyte loss may boost secondary bacterial infections that may be polymicrobial, adding more serious and bad outcomes of the disease [10].

Cytokines are increasingly produced, mainly IL- $1 \beta$, IL-2, IL-6, IL-10, interferon-inducible protein-10 (IP10), IFN- $\gamma$, and TNF- $\alpha$ in severe cases [11]; this cytokine storming can initiate inflammatory-triggered multi-organ dysfunction, mainly adult respiratory distress syndrome (ARDS), respiratory failure, hepatic, renal and myocardial injury with elevated corresponding biochemical markers [12].

SARS-CoV-2-specific antibodies (IgM and $\mathrm{IgG}$ ) can be detected in the first week of illness in less than $40 \%$, which may approach $100 \%$ after 2 weeks of the onset of infection [13].

Patients who are susceptible to serious respiratory complications, mainly pneumonia and its sequelae as hospitalization and death, are the elderly, patients with chronic diseases as chronic obstructive airway disease, ischemic heart disease, diabetes mellitus, chronic liver disease, and pregnant women, so they have the highest priority of vaccination [14].

Vaccines that improve the immunological integrity of the respiratory system, such as Inactivated Influenza Vaccine Trivalent Types A and B (Split Virion) [VAXIGRIP ${ }^{\circledR}$ ] containing three strains of influenza virus developed by culture on embryonic eggs [15].

It has been shown to induce specific IgG antibodies to $\mathrm{H} 1 \mathrm{~N} 1$ and H3N2 influenza viruses, respectively, with a substantial improvement in post-vaccination B cell counts; influenza vaccination should be given annually as immunity diminishes over the next year [16].

Vaccination may be suboptimal in the elderly due to a progressive decrease in humoral and cell-mediated immunity, aging of the immune system with a significant reduction in the efficacy of natural killer (NK) cells, defective differentiation, and performance of $\mathrm{B}$ and $\mathrm{T}$ lymphocytes [17, 18].

Pneumococcal conjugate vaccine (PCV) which is composed of pneumococcal capsular polysaccharides covalently bound to a protein that is almost identical to diphtheria toxin (cross-reactive material 197 [CRM197]; a non-toxic form of diphtheria toxoid) which enhances the conversion of T-cellindependent free-polysaccharide immune response to T-celldependent immune response [19] leading to maturation of B cell response and the development of B cell memory [20].

Conjugated polysaccharides stimulate mucosal immunity that eradicates the nasopharyngeal reservoir. Prevnar13 contains 13 capsular types; it is given as $0.5 \mathrm{ml}$ intramuscularly [21, 22].

Prevnar $13^{\circledR}$ vaccine has been indicated for adults with the risk of invasive pneumococcal infection with extended efficacy to protect from bacteremia, meningitis, and acute otitis media. Influenza vaccine can be administered with pneumococcal polysaccharide vaccine using separate syringes at different injection sites [23, 24].

In diabetes mellitus, protective vaccines are recommended due to the increased risk of drug-resistant influenza and pneumococcal disease, especially if the patients are older with decreased immunity and impaired respiratory function, confirming the importance of immunization; it was recommended that influenza and pneumococcal vaccines should be given for all diabetic patients [25].

In the current study, we retrospectively evaluated the impact of routine and regular yearly administration of recombinant influenza and single administration of Prevnar13 vaccines in high-risk patients with type 2 diabetes mellitus on the occurrence of serious respiratory infection including COVID-19.

\section{Research design and methods}

\section{1-Patient selection}

This is a retrospective and observational study from January 2016 to September 2020; the clinical data of 100 diabetic patients were retrospectively evaluated (Fig. 1); they were accustomed to receive regularly and yearly Vaxigrip ${ }^{\circledR}$ [Inactivated Influenza Vaccine Trivalent Types: 2 type A and 1 type B], $0.5 \mathrm{ml}$ in the deltoid region, together with Prevnar $13^{\circledR}$ vaccine once every $3-5$ years, $0.5 \mathrm{ml}$ intramuscular in the other deltoid region.

\section{Sample size}

The internal medicine department serves nearly 1000 patients per year (3000 over the period of the study), and nearly $25 \%$ of them present with repeated respiratory tract 


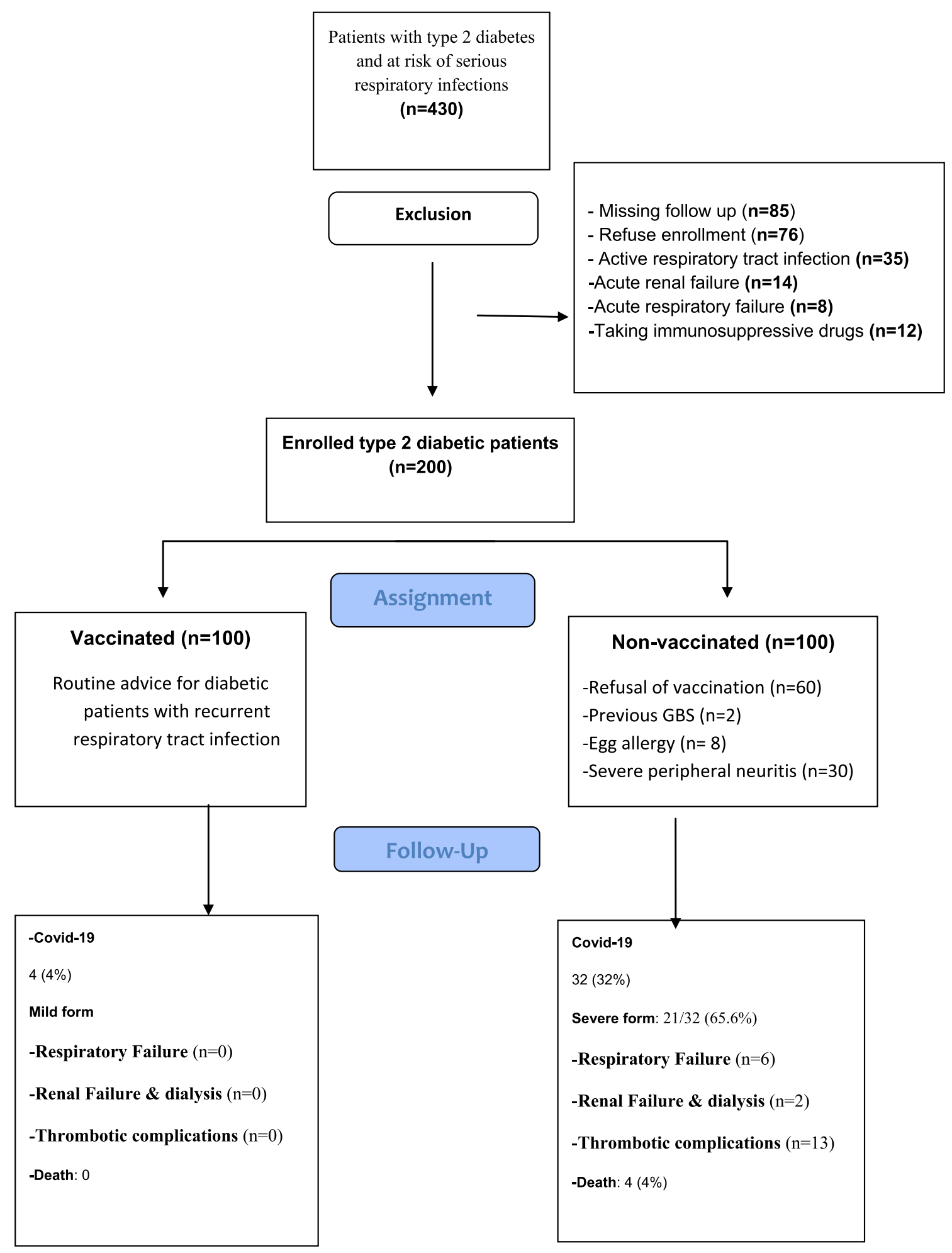

Fig. 1 Flowchart of the studied groups

infection, so with a margin of error of $9.78 \%$, a confidence level of $95 \%$, a response distribution of $50 \%$, and a power of $80 \%$, the recommended sample size was at least 100 patients in each arm with $p<0.05$.

\section{Inclusion criteria}

Patients with type 2 diabetes and at risk of serious respiratory infections, mainly elderly, are included; other comorbidities 
include ischemic heart disease, bronchial asthma, and renal failure; also, patients with recurrent seasonal influenza and recurrent trachea-bronchitis were included.

Patients with a previous history of allergy to the utilized vaccines, egg allergy, Guillain-Barré syndrome, current active upper respiratory tract infection, and severe peripheral neuritis were excluded.

As a routine step in the care of these patients, we advised them to receive these vaccines and oral consent was obtained from all patients to share their data in this research.

\section{Control group}

One hundred type 2 diabetic patients who fulfilled the inclusion criteria but did not receive both studied vaccines due to refusal of vaccination $(n=60)$, previous Guillain-Barré syndrome $(n=2)$, egg allergy $(n=8)$, and severe peripheral neuritis $(n=30)$ were matched for age and sex and shared a similar exposure.

Patients were evaluated by:

- Comprehensive clinical examination, including vital signs and clinical characteristics of renal, heart, or respiratory diseases, had been reported.

- Features of COVID-19: Mild COVID-19 is manifested by fever, malaise, cough, headache, myalgia, and diarrhea, loss of smell and taste without dyspnea, or abnormal chest radiology. Moderate COVID-19 is manifested as any symptom of the mild disease and lung ultrasound and radiology features with oxygen saturation (SpO2) $\geq 94 \%$ on room air. Severe COVID-19 presented with $\mathrm{SpO} 2<94 \%$ on room air, a ratio of arterial partial pressure of oxygen to fraction of inspired oxygen $(\mathrm{PaO} 2 /$ FiO2) $<300 \mathrm{~mm} \mathrm{Hg}$, respiratory rate $>30 / \mathrm{min}$, or lung affection $>50 \%$. Critical COVID-19 is characterized by respiratory failure, septic shock, and/or multiple organ failure [26].

- Airflow screening questionnaire (Fig. 2) [27] was evaluated before vaccination and every 6 months post-vaccination with comparison; a score $>25$ implied obstruction of airflow.

- Routine laboratory investigations included liver function tests, coagulation profile, renal function tests, complete blood count, $\mathrm{C}$ reactive protein, and $\mathrm{D}$ dimer level.

- PCR for the nasopharyngeal swab of the suspected cases

Well-trained personnel had performed the sampling. The positive results suggested SARS-CoV-2 infection, but bacteria and other virus-induced co-infection could not be excluded. The negative results could not exclude SARSCoV-2 infection and should be judged by combining clinical observation; patients who suffered from fever, sore throat, dyspnea, and cough with confirmed exposure to confirmed cases, with typical chest computerized tomography (CT) findings, are highly suggestive of COVID-19 [28].

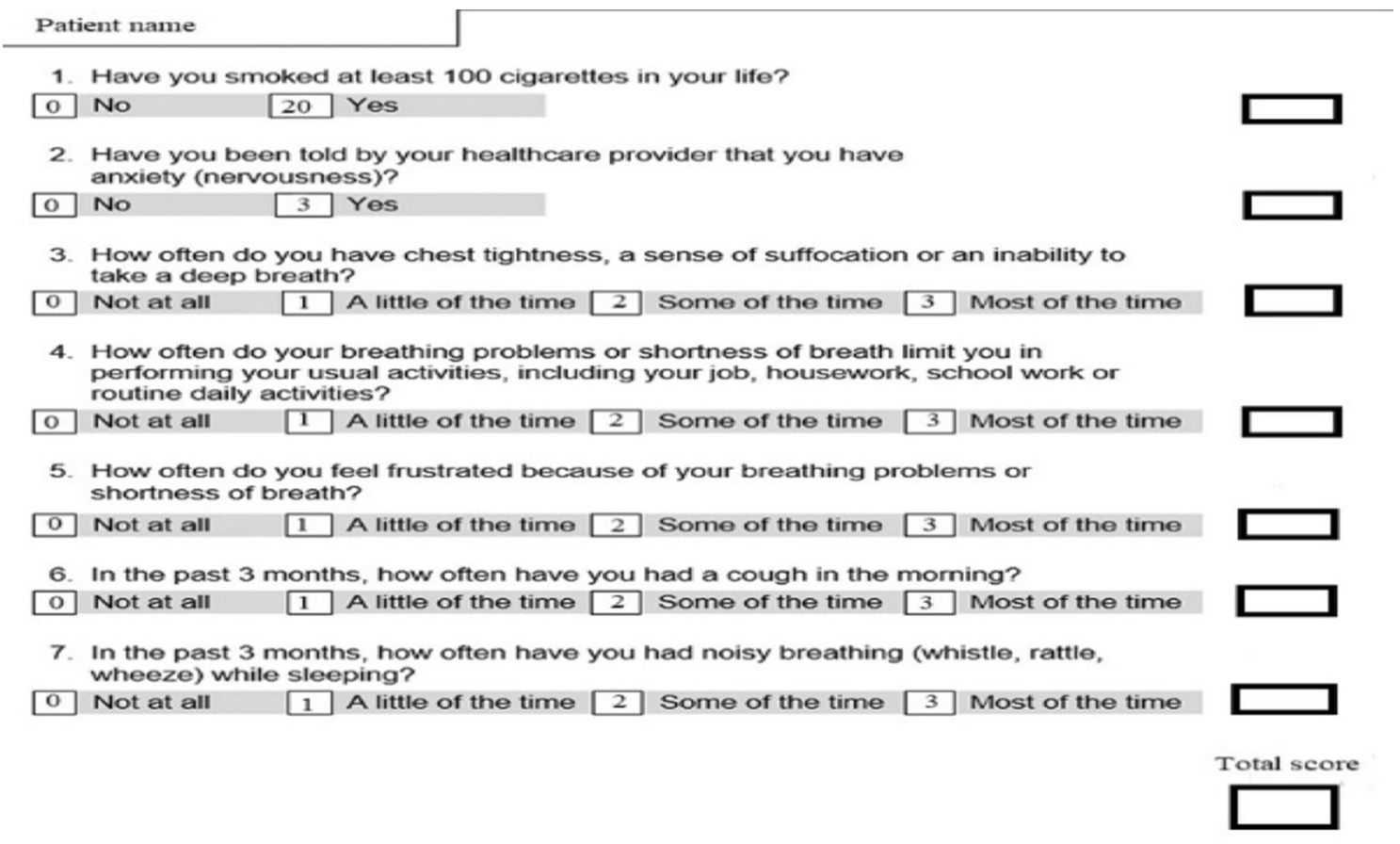

Fig. 2 Airflow screening questionnaire 


\section{Lung ultrasound}

Features highly suggestive of COVID-19 are confluent and isolated B lines, thickened irregular pleural lines, sub-pleural pulmonary consolidations, and air bronchogram [29].

\section{High-resolution CT chest}

Features in CT may show ground-glass opacities, with a rounded morphology and peripheral lung affection [30].

\section{Follow-up}

The first patient had received Prevnar13 in January 2016, and the last patient in October 2019; 15 patients received a second dose of Prevnar 13 after 3 years from the last dose.

The first patient who had received the first dose of Vaxigrip in January 2016 and the last one in October 2019 had been given Vaxigrip yearly; they were followed up after the first dose of vaccines to detect the impact of both vaccines on the occurrence of respiratory complications and on systemic inflammatory parameters by laboratory evaluation every 4 months; they were encouraged to consult us in case of any respiratory distress, and during COVID-19 pandemic they were followed strictly from January 2020 until September 2020; any suspected case was evaluated clinically and by lung ultrasound and then referral for CT chest when indicated.

\section{Study outcome}

- The time from the first recurrence of respiratory tract infection or life-threatening chest infections after vaccination up to death or the end of the study period (September 1, 2020), whichever came first.

- Incidence of COVID-19 and complications including death in the vaccinated patients when compared to the non-vaccinated patients.

\section{Statistical analysis}

All statistical analyses were performed using the SPSS version 20 software (SPSS Inc., Chicago, IL, USA). Shapiro-Wilk test was used to test the distribution of data. Independent samples' Student's t test was used to compare between the study and control groups of normally distributed variables, while Mann-Whitney U test was used for non-normally distributed variables. The chi-square or Fisher's exact tests were used for categorical variables when appropriate. The paired t test was appropriately used. $P$ value $<0.05$ was considered significant. Survival analysis was performed using Kaplan-Meier test; the significance of the curves was assessed using log-rank test. Cox proportional hazard analysis was used to estimate the hazard ratio of COVID19-related adverse events and death.

\section{Results}

One hundred patients had received the combined vaccines [yearly Vaxigrip from 2016 till 2019, 85 patients received Prevnar 13 once and 15 patients received a second dose after 3 years]; all the patients in the study group were diabetic; 34 patients were treated with insulin Mixtard (soluble insulin $30 \%$ and isophane insulin 70\%) $56.3 \pm 23.6$ units daily, 66 patients were on oral hypoglycemic agents (glimepiride $n=39$, glyburide $n=27$, vildagliptin $n=66$ ), and $48 \%$ were smokers. They had received the vaccines due to recurrent bronchopneumonia $(n=39)$, ischemic heart disease $(n=25)$, renal impairment $(n=12)$, and recurrent seasonal influenza $(n=24)$.

They were compared with a control group of patients with type 2 diabetes mellitus who did not receive the vaccines due to their refusal of vaccination, allergic reaction to influenza vaccine or egg allergy, and severe peripheral neuritis for fear of its aggravation. Forty-two patients had recurrent bronchitis, recurrent bronchopneumonia $(n=15)$, ischemic heart disease $(n=26)$, and renal impairment $(n=17)$.

They were treated with insulin Mixtard (soluble insulin 30\% and isophane insulin 70\%) $52.2 \pm 13.5$ units daily $(n=46), 54$ patients were on oral hypoglycemic agents (glimepiride $n=21$, glyburide $n=12$, metformin $n=21$, vildagliptin $n=54$ ), and $44 \%$ were smokers. The baseline laboratory characteristics of both groups are shown in Table 1.

Tables 2 and 3 show the impact of Vaxigrip and Prevnar 13 on the airflow screening questionnaire and laboratory parameters of the studied patients when compared to the control group at the end of follow-up, including the incidence and complications of COVID-19 in both groups.

The patients who were followed up after combined vaccinations showed a significant improvement in respiratory tract symptoms as dyspnea, cough, expectoration, and quality of life guided by a significant decrease in the airflow screening questionnaire ( $14.1 \pm 2.7$ post-vaccination vs. $24.15 \pm 5.43$ pre-vaccination $=0.0001$ ) and highly significant reduction when compared to the control group presented with respiratory symptoms $(14.1 \pm 2.7$ vs. $26.5 \pm 7.45, p=0.0001)$.

In the study group, 12 patients had developed mild upper respiratory tract infection in the form of tracheobronchitis after $18.5 \pm 9.5$ months of follow-up, which responded well to levofloxacin $750 \mathrm{mg}$ for 5 days; however, 17 patients of the control group developed lower respiratory tract infections ( 8 patients needed hospitalization and 9 patients needed $14.5 \pm 3.8$ days of broad spectrum antibiotics $\left(p=0.0005, x^{2}=11.977\right)$. 
Table 1 Baseline characteristics of patients in the study and control groups
Table 2 Impact of Vaxigrip and Prevnar13 on airflow screening questionnaire and laboratory parameters of the studied patients

\begin{tabular}{|c|c|c|c|}
\hline & Control $(n=100)$ & Case $(n=100)$ & $p$ \\
\hline$F / M$ & $28 / 72$ & $22 / 78$ & 0.147 \\
\hline Age & $56.03 \pm 10.9$ & $57.5 \pm 10.5$ & 0.73 \\
\hline BMI $\left(\mathrm{kg} / \mathrm{m}^{2}\right)$ & $31.4 \pm 1.8$ & $31.9 \pm 1.5$ & 0.16 \\
\hline Smoking & $48(48 \%)$ & $44(44 \%)$ & 0.3 \\
\hline Airflow screening questionnaire & $24.15 \pm 5.43$ & $24.53 \pm 6.2$ & 0.196 \\
\hline AST IU/L & $49.3 \pm 9.9$ & $41.9 \pm 10.2$ & 0.86 \\
\hline ALT IU/L & $57.9 \pm 11.1$ & $47.9 \pm 21.8$ & 0.001 \\
\hline Total bilirubin mg/dl & $0.94 \pm 0.41$ & $1.03 \pm 0.36$ & 0.34 \\
\hline Albumin gm/dl & $4.7 \pm 0.42$ & $5.1 \pm 0.24$ & 0.001 \\
\hline INR & $0.93 \pm 0.11$ & $1.09 \pm 0.19$ & 0.1 \\
\hline Creatinine $\mathrm{mg} / \mathrm{dl}$ & $1.156 \pm 0.24$ & $1.17 \pm 0.27$ & 0.234 \\
\hline $\operatorname{WBC}\left(10^{3} / \mathrm{ul}\right)$ & $8.9 \pm 2.1$ & $11.7 \pm 2.08$ & 0.699 \\
\hline lymphocytes $\left(10^{3} / \mathrm{ul}\right)$ & $2.96 \pm 0.72$ & $1.97 \pm 0.62$ & 0.257 \\
\hline NLR & $4.67 \pm 1.3$ & $5.8 \pm 0.95$ & 0.001 \\
\hline Hemoglobin gm/dl & $12.5 \pm 0.98$ & $11.4 \pm 1.33$ & 0.039 \\
\hline Platelet count $\left(10^{3} / \mathrm{ul}\right)$ & $186.9 \pm 12.8$ & $158.6 \pm 26.5$ & 0.001 \\
\hline FBS mg/dl & $197.9 \pm 30.2$ & $208.9 \pm 23.8$ & 0.1 \\
\hline HBA1c $\%$ & $8.9 \pm 0.46$ & $9.4 \pm 0.6$ & 0.11 \\
\hline \multicolumn{4}{|l|}{$E S R$} \\
\hline 1st Hour & $50.9 \pm 11.4$ & $68.2 \pm 22.05$ & 0.001 \\
\hline 2st Hour & $78.2 \pm 9.3$ & $87.2 \pm 27.1$ & 0.001 \\
\hline CRP g/l & $10.1 \pm 1.3$ & $9.6 \pm 2.4$ & 0.0012 \\
\hline D-Dimer (ng/dl) & $344.8+115.4$ & $398.4 \pm 94.1$ & 0.088 \\
\hline
\end{tabular}

INR International normalized ratio; NLR Neutrophil-lymphocyte ratio; CRP C-reactive protein

\begin{tabular}{llcc}
\hline & Post-vaccination & Pre-vaccination & $p$ \\
\hline Airflow screening questionnaire & $24.15 \pm 5.43$ & $14.1 \pm 2.7$ & 0.0001 \\
WBC $\left(10^{3} / \mathrm{ul}\right)$ & $8.9 \pm 2.1$ & $5.64 \pm 0.81$ & 0.0001 \\
Lymphocytes $\left(10^{3} / \mathrm{ul}\right)$ & $2.96 \pm 0.72$ & $3.3 \pm 0.92$ & 0.008 \\
NLR & $4.67 \pm 1.3$ & $1.87 \pm 0.4$ & 0.0001 \\
Platelets $\left(10^{3} / \mathrm{ul}\right)$ & $186.9 \pm 12.8$ & $205 \pm 43.4$ & 0.0001 \\
ESR & & & \\
First hour & $50.9 \pm 11.4$ & $24.1 \pm 9.8$ & 0.001 \\
Second hour & $78.2 \pm 9.3$ & $33.9 \pm 15.2$ & 0.001 \\
CRP g/l & $10.1 \pm 1.3$ & $5.4 \pm 1.27$ & 0.0001 \\
D-Dimer (ng/dl) & $344.8+115.4$ & $158.5 \pm 59.3$ & 0.0001 \\
\hline
\end{tabular}

Four patients (4\%) in the study group had developed mild COVID-19 during the period of follow-up in COVID19 pandemic from January 2020 till September 2020, in the form of low-grade fever and gastrointestinal symptoms (abdominal pain, diarrhea, loss of taste, and smell) without respiratory manifestations; the data were collected by clinical examination of suspected cases and evaluation of COVID-19 questionnaire including high-grade fever, body aches, gastrointestinal symptoms, sense of smell and taste, and respiratory manifestations after being informed to report any suspicious symptoms confirmed by laboratory findings, lung ultrasound, and CT chest.

No recorded complications in the COVID-19-infected patients with a significant reduction in inflammatory parameters as leucocytes count, neutrophil-lymphocyte ratio, platelet count, C-reactive protein, and D-dimer levels when compared to the pre-vaccination values (Table 2).

A significant number (32\%) in the control group had COVID-19 infection with a significant increase in inflammatory and coagulation parameters, which was severe in $21 / 32(65.6 \%)$ patients and complicated with respiratory 
Table 3 Impact of Vaxigrip and Prevnar13 on airflow screening questionnaire and laboratory parameters of the studied patients when compared to control group at the end of follow-up

\begin{tabular}{llll}
\hline & Control group & Study group & $p$ \\
\hline $\begin{array}{l}\text { Airflow screening question- } \\
\quad \text { naire }\end{array}$ & $26.5 \pm 7.45$ & $14.1 \pm 2.7$ & 0.0001 \\
WBC $\left(10^{3} / \mathrm{ul}\right)$ & $16.47 \pm 1.3$ & $5.64 \pm 0.81$ & 0.0002 \\
Lymphocytes $\left(10^{3} / \mathrm{ul}\right)$ & $1.3 \pm 0.34$ & $3.3 \pm 0.92$ & 0.0001 \\
NLR & $8.1 \pm 1.9$ & $1.87 \pm 0.4$ & 0.0001 \\
Platelet count $\left(10^{3} / \mathrm{ul}\right)$ & $122.4 \pm 28.2$ & $205 \pm 43.4$ & 0.001 \\
ESR & & & \\
First hour & $81.3 \pm 24.1$ & $24.1 \pm 9.8$ & 0.0001 \\
Second hour & $101.8 \pm 19.6$ & $33.9 \pm 15.2$ & 0.001 \\
CRP g/l & $35.2 \pm 8.4$ & $5.4 \pm 1.27$ & 0.0001 \\
D-Dimer (ng/dl) & $1288+322.5$ & $158.5 \pm 59.3$ & 0.0001 \\
COVID-19 $(n, \%)$ & $32(32 \%)$ & $4(4 \%)$ & 0.0001 \\
Complications & & & \\
Respiratory failure $(n=6)$ & 6 & 0 & 0.0022 \\
Renal failure and dialysis & 2 & 0 & 0.33 \\
$\quad(n=2)$ & & & 0.00001 \\
Thrombotic complications & 13 & 0 & 0.0286 \\
$\quad(n=13)$ & & 0 & \\
Death $(n=4)$ & 4 & & \\
\hline
\end{tabular}

$N L R$ : Neutrophil-lymphocyte ratio; $C R P$ C-reactive protein

failure in 6 patients, renal failure and dialysis in 2 patients, thrombotic complications in 13 [DVT $(n=3)$, myocardial infarction $(n=5)$, pulmonary embolism $(n=4)$, cavernous sinus thrombosis $(n=1)]$, and death $(n=4)$; lung ultrasound was highly efficient in directing diagnosis and confirmed by high-resolution CT chest (Fig. 3).

The impact of giving vaccination to diabetic patients on survival and occurrence of major events as serious respiratory tract infections including COVID-19 and death was analyzed by Kaplan-Meier survival curve (Fig. 4). The hazard ratio (HR) of getting significant respiratory tract infection was 2.29 higher in the non-vaccinated control group when compared to the vaccinated diabetic patients $(95 \%$ CI: $0.39-4.8, p=0.001$ ); HR of death due to COVID-19 was 10.24 times higher in the non-vaccinated control group when compared to the vaccinated diabetic patients $(95 \% \mathrm{CI}$ : $0.45-21.8, p=0.001$ ).

The severity of COVID-19 in patients with diabetes had been correlated with HBA1C $(r=0.422, p=0.007)$, combined Vaxigrip and Prevnar13 vaccination $(r=-1.12$, $\mathrm{p}=0.0001)$, serum creatinine $(r=0.537, p=0.001)$, neutrophil-lymphocyte ratio $(r=0.597, p=0.001)$, and thrombocytopenia $(r=0.377, p=0.003)$.

\section{Discussion}

Patients with chronic respiratory illnesses have altered lung functions and may have other comorbidities like cardiovascular disease, diabetes, chronic renal failure, and malignant conditions, rendering these patients highly vulnerable to viral or bacterial infection [30].

Prevnar13 had been recommended for all adults who are at high risk of respiratory tract infection, with underlying medical conditions or immunosuppression in more than 25 countries [31]. The efficacy of the pneumococcal vaccine in diabetic patients might reach $84 \%$ and does not decline with increasing duration after vaccination. The vaccine was highly cost-effective in preventing pneumococcal infection in older patients [32]; it was safe, with enhanced immunogenicity [33].

Influenza vaccination in patients with diabetes enhances B-lymphocytes function and antibody production, leading to a reduction in hospitalizations due to complications and the rate of deaths in more than half of the cases [34].

A large study of mortality in COVID-19 had revealed that poor glycemic control (HbA1c more than 10\%) before hospital admission was associated with a higher risk of in-hospital death compared with those with HbA1c of less 6.5\% [35].

Hyperglycemia at admission was associated with radiological severity, worse outcome including ICU admission, mechanical ventilation, or death, when compared with patients with normal glucose level, denoting that improving glycemic control is the most important target whether the patient is diabetic or not [36].

The mortality was significantly higher in patients with diabetes or uncontrolled hyperglycemia during their hospital stay (30\%) than those with euglycemia (6\%), and these patients had higher rates of neutrophilia, lymphopenia, higher C-reactive protein, and pro-calcitonin with higher risks for diabetic ketoacidosis and hyperglycemic hyperosmolar syndrome [37]; this rapid metabolic deterioration could be explained by marked affection of insulin-secreting ability of the pancreas, together with the metabolic paralysis mediated by the cytokine release, viral affinity to ACE2 receptors in the pancreatic $\beta$-cells, and anorexia that prevent regular administration of anti-diabetic drugs $[38,39]$.

The current study assessed the impact of combined influenza and Prevnar13 vaccination in diabetic patients with recurrent respiratory tract infections on systemic inflammation, and the possible protection from COVID-19; it had revealed that the combination of both vaccines had significantly improved respiratory symptoms, with marked improvement in systemic inflammation parameters when evaluated regularly during the period of follow-up.

It was suggested that both vaccines might improve the outcomes in the form of significant reductions in numbers 
Fig. 3 Lung ultrasound with corresponding computed tomography of the chest, A: normal lungs with horizontal A lines, B: normal CT chest, C: sub-pleural pulmonary consolidation $2.7 \mathrm{~cm}$ with isolated B lines, D: CT chest showed ground-glass opacities
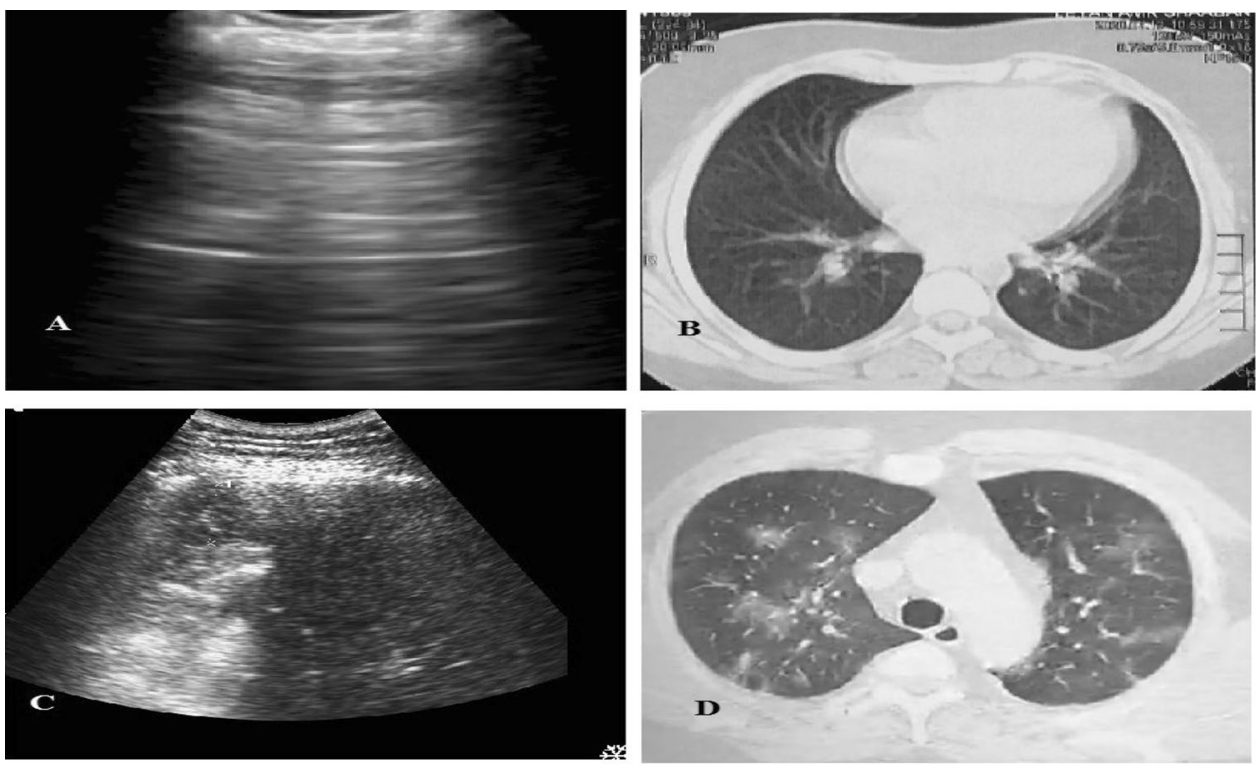
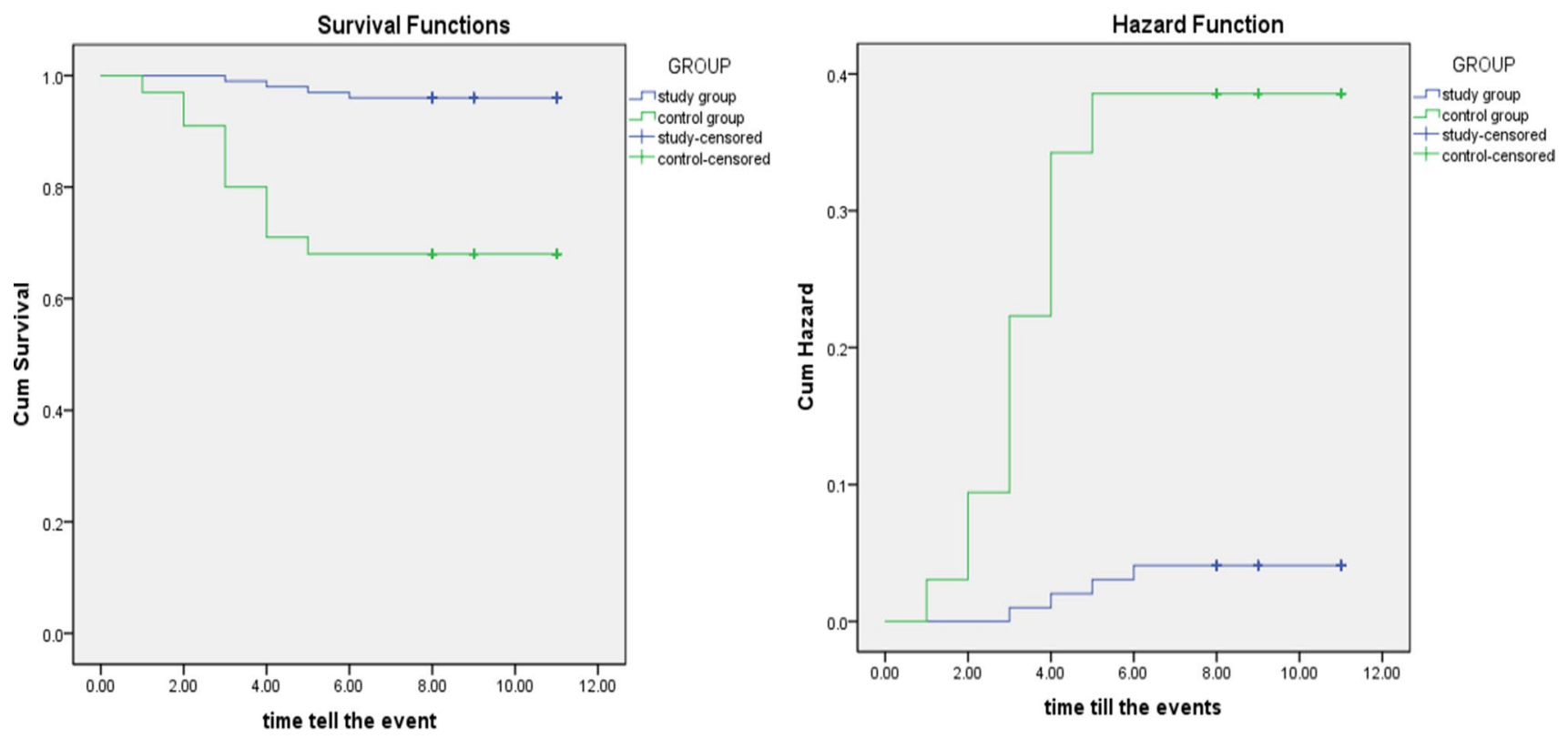

Fig. 4 The impact of adding combined influenza and pneumococcal conjugate vaccines on risk of serious respiratory infections including COVID-19 in the treated and control patients

of patients presented with bronchopneumonia which was mild infection that responded to medications in a short period of time; however, $17 \%$ of patients in the control group developed significant lower respiratory tract infections that needed hospitalization in 8 patients $(p=0.0005$, $x^{2}=11.977$ ).

Our results were supported by the fact that influenza vaccine caused a significant reduction in rate of pneumonia (32\%), hospitalization, and the risk of death by $50 \%$ [40] and also reduced the morbidity and mortality in patients with cardiac disease in $37 \%$ [40].
The Community-Acquired Pneumonia Immunization Trial in Adults (CAPITA) had shown the effectiveness of the pneumococcal conjugate vaccine in older patients, and data had indicated that the pneumococcal and influenza vaccines might reduce community-acquired pneumonia and acute exacerbations in patients with the chronic respiratory disease [41-43].

Four (4\%) patients in the study group had developed mild COVID-19, mainly gastrointestinal, without respiratory manifestations with no recorded complications and a significant reduction in inflammatory markers; however, 
significant number (21\%) in the control group had severe COVID-19 infection with life-threatening complications and death in 4 patients, reflecting the potential protective role of combining both studied vaccines against COVID19 , both groups had the same environmental exposure and the same preventive measures and comorbid conditions.

In agreement with our results is the concept that influenza and pneumococcal vaccines can prevent the numbers of pneumococcal and influenza hospital admissions, reduces the needed care for non-COVID-19 patients, and minimizes possible superadded infections, preventing a considerable amount of COVID-19-related mortality [44], and this is supported by the fact that there is a lower rate of SARS-CoV-2 in children who were infected with influenza or had been vaccinated [45].

The extended protection of both studied vaccines can be explained by direct stimulation of Toll-like receptor (TLR), eliciting both humoral and $\mathrm{T}$ cell responses, production of specific natural killer (NK) cells, with enhanced long-term memory, and possible activation of OX 40 and OX40L [46, 47].

The limitations of the current study are its retrospective evaluation and the limited information about the other treatments that the patients received in both the vaccination and control group. In addition, there is a lack of practical methods to measure the antibody titers of both vaccines. Further, the detailed analysis of some essential risk factors of type 2 diabetes as physical inactivity and dietary habits was deficient as there was a shortage of such data in the obtained medical records. We think that the study sample size might not be large enough to reflect more accurate results regarding this issue. All patients were selected from only one, albeit large, hospital. Further future randomized control trials are required to study these associations and confirm them and could build upon these retrospective data from our study. Randomized control trials could establish direct causation more reliably.

Acknowledgements Amr Hanafy is the guarantor of this work, had full access to all the data in the study, and takes responsibility for the integrity of the data and the accuracy of the data analysis. Amr Hanafy, Waseem M. Seleem, and Hany A. Elkattawy had followed-up the patients clinically, Amr Hanafy performed lung ultrasound and statistical analysis and wrote the manuscript, all authors had revised the manuscript. The authors deeply thank the Researchers Supporting Program (TUMA-Project-2021-35), Almaarefa University, Riyadh, Saudi Arabia for supporting the efforts of researchers in this work.

Funding No funding.

\section{Declarations}

Conflict of interest The authors that they have declare no conflict of interest.

\section{References}

1. Nichol KL. Heterogeneity of influenza case definitions and implications for interpreting and comparing study results. Vaccine. 2006;24(44-46):6726-8.

2. Channappanavar R, Zhao J, Perlman S. T cell-mediated immune response to respiratory coronaviruses. Immunol Res. 2014;59(1-3):118-28.

3. Lippi G, Plebani M. Laboratory abnormalities in patients with COVID-2019 infection. Clin Chem Lab Med. 2020;58(7):1131-4.

4. Qin C, Zhou L, Hu Z, Zhang S, Yang S, Tao Y, et al. Dysregulation of immune response in patients with coronavirus 2019 (COVID19) in Wuhan, China. Clin Infect Dis. 2020;71(15):762-8.

5. Moon C. Fighting COVID-19 exhausts T cells. Nat Rev Immunol. 2020;20(5):277.

6. Li D, Chen Y, Liu H, Jia Y, Li F, Wang W, et al. Immune dysfunction leads to mortality and organ injury in patients with COVID19 in China: insights from ERS-COVID-19 study. Signal Transduct Target Ther. 2020;5:62.

7. Liana L, Linga $X$, Chen L. T cell response in patients with COVID-19. Blood Sci. 2020;2(3):76-8.

8. Kopf M, Ruedl C, Schmitz N, Gallimore A, Lefrang K, Ecabert $\mathrm{B}$, et al. OX40-deficient mice are defective in Th cell proliferation but are competent in generating B cell and CTL Responses after virus infection. Immunity. 1999;11(6):699-708.

9. Zheng HY, Zhang M, Yang CX, Zhang N, Wang XW, Yang XP, et al. Elevated exhaustion levels and reduced functional diversity of $\mathrm{T}$ cells in peripheral blood may predict severe progression in COVID-19 patients. Cell Mol Immunol. 2020;17(5):541-3.

10. Chen N, Zhou M, Dong X, Qu J, Gong F, Han Y, et al. Epidemiological and clinical characteristics of 99 cases of 2019 novel coronavirus pneumonia in Wuhan, China: a descriptive study. Lancet. 2020;395(10223):507-13.

11. Yang L, Gou J, Gao J, Huang L, Zhu Z, Ji S, et al. Immune characteristics of severe and critical COVID-19 patients. Signal Transduct Target Ther. 2020;5(1):179.

12. Yang L, Liu S, Liu J, Zhang Z, Wan X, Huang B, et al. COVID-19: immunopathogenesis and immunotherapeutics. Signal Transduct Target Ther. 2020;5:128.

13. Diao B, Wang C, Tan Y, Chen X, Liu Y, Ning L, et al. Reduction and functional exhaustion of T cells in patients with coronavirus disease 2019 (COVID-19). Front Immunol. 2020;1(11):827.

14. Heo JY, Song JY, Noh JY, Choi MJ, Yoon JG, Lee SN, et al. Effects of influenza immunization on pneumonia in the elderly. Hum Vaccin Immunother. 2018;14(3):744-9.

15. National Advisory Committee on Immunization (NACI). Statement on seasonal influenza vaccine for 2013-2014. Can Commun Dis Rep 2013;39(ACS-4):1-37.

16. Lambert ND, Ovsyannikova IG, Pankratz VS, Jacobson RM, Poland GA. Understanding the immune response to seasonal influenza vaccination in older adults: a systems biology approach. Expert Rev Vaccines. 2012;11(8):985-94.

17. Beyer WE, McElhaney J, Smith DJ, Monto AS, Nguyen-VanTam JS, Osterhaus AD. Cochrane re-arranged: support for policies to vaccinate elderly people against influenza. Vaccine. 2013;31(50):6030-3.

18. Frasca D, Riley RL, Blomberg BB. Humoral immune response and B-cell functions including immunoglobulin class switch are down regulated in aged mice and humans. Semin Immunol. 2005; 17(5):378-84.

19. Juergens C, de Villiers PJ, Moodley K, Jayawardene D, Jansen KU, Scott DA, et al. Safety and immunogenicity of 13-valent pneumococcal conjugate vaccine formulations with and without aluminum phosphate and comparison of the formulation of choice with 23 -valent pneumococcal polysaccharide vaccine in elderly 
adults: a randomized open-label trial. Hum Vaccin Immunother. 2014;10(5):1343-53.

20. Clutterbuck EA, Lazarus R, Yu LM, Bowman J, Bateman EA, Diggle L, et al. Pneumococcal conjugate and plain polysaccharide vaccines have divergent effects on antigen-specific B cells. J Infect Dis. 2012;205(9):1408-16.

21. Bonten MJ, Huijts SM, Bolkenbaas M, Webber C, Patterson S, Gaultet $\mathrm{S}$, et al. Polysaccharide conjugate vaccine against pneumococcal pneumonia in adults. N Engl J Med. 2015;372:1114.

22. Musher DM. Editorial commentary: should 13-valent proteinconjugate pneumococcal vaccine be used routinely in adults? Clin Infect Dis. 2012;55:265.

23. Weiss S, Falkenhorst G, van der Linden M, Imöhl M, von Kries R. Impact of 10- and 13-valent pneumococcal conjugate vaccines on incidence of invasive pneumococcal disease in children aged under 16 years in Germany, 2009 to 2012. Euro Surveill 2015;20(10):21057.

24. Grilli G, Fuiano L, Biasio LR, Pregliasco F, Plebani A, Leibovitz $\mathrm{M}$, et al. Simultaneous influenza and pneumococcal vaccination in elderly individuals. Eur J Epidemiol. 1997;13(3):287-91.

25. Recommended adult immunization schedule-United States. Centers for disease control and prevention. J Midwifery Womens Health. 2012; 57(2):188-95.

26. Wu Z, McGoogan JM. Characteristics of and important lessons from the coronavirus disease 2019 (COVID-19) outbreak in China: summary of a report of 72314 cases from the Chinese center for disease control and prevention. JAMA. 2020;323(13):1239-42.

27. Sogbetun F, Eschenbacher WL, Welge JA, Panos RJ. Veterans airflow obstruction screening questionnaire: a survey to identify veterans with airflow obstruction. Chronic Obstr Pulm Dis. 2016;3(4):705-15

28. Xie X, Zhong Z, Zhao W, Zheng C, Wang F, Liu J. Chest CT for typical 2019-nCoV pneumonia: relationship to negative RT-PCR testing. Radiology. 2020;296(2):E41-5.

29. Fiala MJ. Ultrasound in COVID-19: a timeline of ultrasound findings in relation to CT. Clin Radiol. 2020;75(7):553-4.

30. Chung M, Bernheim A, Mei X, Zhang N, Huang M, Zeng X, et al. CT imaging features of 2019 novel Coronavirus (2019-nCoV). Radiology. 2020;295(1):202-7.

31. Divo M, Cote C, de Torres JP, Casanova C, Marin JM, PintoPlata V, et al. Co-morbidities and risk of mortality in patients with chronic obstructive pulmonary disease. Am J Respir Crit Care Med. 2012;186(2):155-61.

32. Esposito S, Bonanni P, Maggi S, Tan L, Ansaldi F, Lopalco PL, et al. Recommended immunization schedules for adults: Clinical practice guidelines by the Escmid Vaccine Study Group (EVASG), European Geriatric Medicine Society (EUGMS) and the World Association for Infectious Diseases and Immunological Disorders (WAidid). Hum Vaccin Immunother. 2016;12(7):1777-94

33. Jackson LA, Neuzil KM, Yu O, Benson P, Barlow WE, Adams $\mathrm{AL}$, et al. Effectiveness of pneumococcal polysaccharide vaccine in older adults. N Engl J Med. 2003;348:1747-55.

34. Ortqvist A. Pneumococcal vaccination: current and future issues. Eur Respir. 2001;18:184-95.

35. Looijmans-Van den Akker I, Verheij TJ, Buskens E, Nichol KL, Rutten GE, Hak E. Clinical effectiveness of first and repeat influenza vaccination in adult and elderly diabetic patients. Diabetes Care. 2006;29:1771-6.

36. Holman N, Knighton P, Kar P, O'Keefe J, Curley M, Weaver A, et al. Risk factors for COVID-19-related mortality in people with type 1 and type 2 diabetes in England: a population-based cohort study. Lancet Diabetes Endocrinol. 2020;8(10):823-833.

37. Li H, Tian S, Chen T, Cui Z, Shi N, Zhong X, et al. Newly diagnosed diabetes is associated with a higher risk of mortality than known diabetes in hospitalized patients with COVID-19. Diabetes Obes Metab. 2020. https://doi.org/10.1111/dom.14099.

38. Kim NY, Ha E, Moon JS, Lee Y-HH, Choi EY. Acute hyperglycemic crises with coronavirus disease-19: case reports. Diabetes Metab. 2020;44:349-53.

39. Rayman G, Lumb A, Kennon B, Cottrell C, Nagi D, Page E, et al. Guidance on the management of diabetic ketoacidosis in the exceptional circumstances of the COVID-19 pandemic. Diabet Med. 2020;37:1214-6.

40. Thompson DL, Jungk J, Hancock E, Smelser C, Landen M, Nichols M, et al. Risk factors for 2009 pandemic influenza A (H1N1)related hospitalization and death among racial/ethnic groups in New Mexico. Am J Public Health. 2011;101:1776-84.

41. Diego C, Vila-Córcoles A, Ochoa O, Rodriguez-Blanco T, Salsench E, Hospital I, et al. Effects of annual influenza vaccination on winter mortality in elderly people with chronic heart disease. Eur Heart J. 2009;30:209-16.

42. Schwarz TF, Flamaing J, Rumke HC, Penzes J, Juergens C, Wenz A, et al. A randomized, double-blind trial to evaluate immunogenicity and safety of 13 -valent pneumococcal conjugate vaccine given concomitantly with trivalent influenza vaccine in adults aged $\geq 65$ years. Vaccine. 2011;29(32):5195-202.

43. Frenck RW Jr, Gurtman A, Rubino J, Smith W, van Cleeff M, Jayawardene D, et al. Randomized, controlled trial of a 13-valent pneumococcal conjugate vaccine administered concomitantly with an influenza vaccine in healthy adults. Clin Vaccine Immunol. 2012;19(8):1296-303.

44. Blasi F, Aliberti S, Bonanni P, Mantero M, Odone A, Signorelli C. Pneumococcal vaccination in adults: recommendations from the Italian Society of Respiratory Medicine (SIMeR) and the Italian Society of Hygiene, Preventive Medicine and Public Health (SItI). Epidemiol Prev. 2014;38(6 Suppl 2):147-51.

45. Thindwa D, Garcia Quesada M, Liu Y, Bennett J, Cohen C, Knoll $\mathrm{MD}$, et al. Use of seasonal influenza and pneumococcal polysaccharide vaccines in older adults to reduce COVID-19 mortality. Vaccine. 2020;38(34):5398-401.

46. Kumar V. Influenza in children. Indian J Pediatr. 2017;84(2):139-43.

47. Goulding J, Tahiliani V, Salek-Ardakani S. OX40:OX40L axis: emerging targets for improving poxvirus-based CD8 (+) T-cell vaccines against respiratory viruses. Immunol Rev. 2011;244(1):149-68.

Publisher's Note Springer Nature remains neutral with regard to jurisdictional claims in published maps and institutional affiliations. 